\title{
Increased longevity of wireless Ad hoc network through fuzzy system
}

\author{
Fawzia Abdali Larki $^{\mathbf{a}^{*}}$, Seyed Javad Mirabedini ${ }^{\mathbf{b}}$ and Ali Harounabadi ${ }^{\mathbf{b}}$
}

\begin{abstract}
${ }^{a}$ Department of Computer, Science and Research of Bushehr Branch, Islamic Azad University, Bushehr, Iran ${ }^{b}$ Department of Computer, Central Tehran Branch, Islamic Azad University, Tehran, Iran

\section{H R O N I C L E \\ A B S T R A C T}

Article history:

Received October 15, 2014

Accepted January 12, 2014

Available online

January 242014

Keywords:

Wireless Ad hoc network

Fuzzy System

Energy

AOMDV Protocol

Routing Algorithms

The Ad hoc network is one of the multistep-based self-organizing networks, which are dynamically changing and are taken more into account as the ways of connecting the terminals through the development of wireless communication terminals. We are faced with numerous challenges in designing a wireless network such as the dynamic topology, common and limited bandwidth, and the limited energy. The nodes are moving according to the continuous changes in the topology and the source-to-destination paths are completely broken. Therefore, the repeated route discovery enhances the delay and overload of routing. Thus, it is essential to consider the link stability while designing the path in order to choose the routing protocol. Providing the multiple paths may lead to the better performance than a path. The transmission energy control in the wireless Ad hoc networks is the option for the level of transmission energy in order to transmit each node packet in this system. Therefore, transmission energy control affects the wireless medium interface. Because of choosing the appropriate protocol, the routing operation can be improved and the energy consumption can be controlled properly as well as enhancing the durability and longevity of network. The main objective of this study is to enhance the network longevity. The proposed algorithm in this research considers the combination of 2 parameters including the rate of node energy and number of steps in Fuzzy System applied on AOMDV Protocol, which is a Multipath Routing Protocol. The results of simulation also indicate the improved performance of proposed algorithm (AOMDV-F) compared to AODV and AOMDV Protocols in NS2 simulator.
\end{abstract}

\section{Introduction}

During the past two decades, there have been tremendous changes on telecommunication industry specially in wireless transportation terminals. The wireless Ad hoc networks have been considered as a way for providing the communication among the terminals without supporting the infrastructures in recent years. One of the advantages of this system is that the communication is flexible under this communication technique. The transfer energy control in wireless Ad hoc networks is an option of

\footnotetext{
* Corresponding author.

E-mail addresses: foziyehabdali@gmail.com (F. Abdali) 
transfer energy level for packet transfer of each node in such system. The transfer energy control in this technique can determine a set of candidate nodes for choosing the next node in routing protocols. It can perform the operation of influencing the traffic control at the transfer layer by impacting on the traffic level associated with the wireless medium. The transfer energy control is the primary factor for determining the multiple measures such as the delay of throughput and energy efficiency.

The wireless Ad hoc networks are the instantaneous or temporary networks generated for a special reason. In fact, the wireless networks carry the mobile nodes. Based on the significant different of ad hoc and conventional wireless networks 802.11, the mobile nodes in the wireless networks are connected with each other without any central infrastructures, access points, or base station to transfer the data at a specified interval. The transfer energy control in this method determines a set of candidate nodes to choose the next node in the routing protocols and to perform the operation of influencing the traffic control at the transfer layer by influencing on the traffic level associated with the wireless medium.

The reduced transfer energy level decreases the energy consumption for communication, increases the reuse of wireless medium and improves the power of wireless networks. On the other hand, the increased transfer energy level is capable of enhancing the scope of transfer nodes. In other words, it reduces the hop count required in each path on average. The sum of transfer delays along the path is reduced in this regard. Furthermore, the higher level of transfer energy improves the signal quality from the receiver to prevent any possible error in the packets as well as delays due to the retransmission of linkage layer. This study tries to improve and control the energy consumption by improving the routing operation, which leads to the enhanced longevity of network. In this method, two parameters (hop count and energy) are combined based on fuzzy logic such that the delay of packet transmission is reduced and the throughput is increased. There are several protocols available for routing in the wireless networks such as aodv, aomdv, etc. However, our proposed method considers the protocol aomdv according to its priorities to other routing protocols. One of the most important priorities is that this protocol is a multipath routing protocol. In other words, it suggests several paths rather than a path at the end of operation and then it provides the most efficient path among several paths after applying the energy on this protocol. This routing improvement operation is shown on AOMDV Protocol. This study tries to reduce the End-to-End Delay in transmitting the packet data and increases the throughput and Packet delivery ratio (PDR) through the wireless networks.

This paper is organized as follows. The second section provides the presented hypotheses; third section describes the related activities and presented algorithms; fourth section provides the proposed framework for acquiring the objective. Fifth section introduces the simulation tools and evaluates the efficiency of proposed method and then we conclude at the end.

\section{Literature review}

Soo et al. (2011) argued that AOMDV protocol was a version beyond AODV routing protocol and it is a type of demand-based protocols. According to the priority of AOMDV to AODV, it significantly reduces the overload due to routing because it introduces and calculates several active paths individually during a path discovery phase. Therefore, it reduces the frequency of route discovery. Similar to AODV, during the route discovery process, this protocol saves the path, in which the source node disseminates the path request to other nodes by that packet, for supporting.

Like AODV, which saves a list of paths in routing table, this protocol assigns a sequence number to each discovered path to the destination. When one of the active paths in AOMDV fails, it selects one of the alternative paths from routing list. AOMDV is implemented based on two characteristics of non-shared link and node. When the path request packet is disseminated, every path request should 
receive the first non-shared node distinct from the destination node. The information of first node is important because the first node carries a list of information for the path request packet. When two or more numbers of path request meet other nodes from the first node distinct from the source node, only the first received path request is disseminated until reaching the destination. On the other hand, this protocol ignores the path request resulted from the first hop of source node and all destination responses resulted from all requests from the requests which are received by other neighboring nodes of destination. The path maintenance process in AOMDV is the same as AODV protocol; considering this fact, the indistinguishable destination is introduced when all listed paths in routing table fail.

Haque and Assi (2007) argued that since each node in the local routing algorithms disseminates a response based on its own position, neighbors and destination, OLEAR Algorithm (Optimal Localized Energy Aware Routing) is introduced. This algorithm moves ahead and chooses a neighbor, which is the closest node to the destination. If there is no neighbor, the algorithm changes the location for improvement. In this case, the neighbors of current node are classified into the top and bottom groups. Then the next node is selected from the bottom group, so that the angle of that node with the current and destination nodes is minimized. If the distance of next node to the destination is lower than the local situation, the algorithm is displaced to forward-moving position. It is not combined with the forward-moving strategies in this algorithm, but in general, the performance of this algorithm on the lifetime optimization of network and energy distribution is maximized. According to the weakness of this method, the performance of this algorithm is not investigated for different topology.

Lai et al. (2012) introduced HEB plan (Hop-aware and Energy-based Buffer management scheme) which is compared with HAB (Hop-aware Buffer management scheme). According to the proposed technique, the buffer is classified into the real time and not real time partitions. A number of hops are considered along the path and the transfer energy levels, and then the number of remaining hops and waiting time are predicted in buffer for determining the priority of transfer packet. In general, the presented approach in this paper is the queue management scheduling algorithm to reduce the likelihood of transmission due to the lack of packet and it operates in an ad hoc network. The proposed algorithm manages the queue (first input first output) to resolve the problem of paths with long hops through the transfer energy levels of hops. The transfer rate and packet loss rate are reduced in this algorithms, thus the consumed energy consumption and End-to-End Delay are also reduced. This algorithm prevents the dissuasion of packets, which have passed a long time in the buffer and have the older ages and also transfer them. According to the weakness of this method, there is no guarantee that the other queue scheduling techniques will achieve such these results. The structures of both protocols are shown in Table 1 and Table 2.

\section{Table 1}

AODV protocol structure

\begin{tabular}{|l|l|l|l|l|} 
End time & Previous hop & Hop count & Number of sequences & Destination
\end{tabular}

Table 2

Structure of AOMDV Protocol

\begin{tabular}{|c|c|c|c|c|c|c|}
\hline \multicolumn{4}{|c|}{ Path List } & \multirow{2}{*}{$\begin{array}{l}\text { Hop } \\
\text { count }\end{array}$} & \multirow{2}{*}{$\begin{array}{c}\text { Number of } \\
\text { sequences }\end{array}$} & Destination \\
\hline End Time 1 & Hop count 1 & Last hop 1 & Forward hop 1 & & & \\
\hline End time 2 & $\begin{array}{c}\text { Hop count } 2 \\
\ldots \ldots . .\end{array}$ & $\begin{array}{c}\text { Last hop } 2 \\
\ldots \ldots . .\end{array}$ & $\begin{array}{c}\text { Forward hop } 2 \\
\ldots . . . .\end{array}$ & & & \\
\hline
\end{tabular}

Bhavsar et al. (2012) compared the operation of 2 types of AODV protocol which sought to find a stable path and AOMDV and introduced the multiple paths according to the sustainability improvement in Mobile Adhoc Network. The results indicated that the AOMDV could significantly reduce the End-to-End Delay. Because of multiple paths, AOMDV can be changed to one of the 
supporting paths when one of active paths is broken. This will finally reduce the route discovery delay. AOMDV needs to disseminate the path request message inclusively in the network in order to find a multiple path in a single route discovery process, thus the routing overload will be increased in the network. On the other hand, AODV removes the unnecessary request packets in both models and this will reduce the routing overload in the network. Given the routing overload in both of them, AOMDV achieves better packet delivery rate and throughput with the stable path than AOMDV. The End-to-End Delay in AOMDV is reduced with the greater extent than AODV, but if considering the issue from the routing overload view, the routing overload can be reduced in the network by choosing AODV for a stable path. Biradar et al. (2010) evaluated the operation of AODV and AOMDV. The comparison is based on the packet delivery rate, routing overload, the average End-to-End Delay and number of lost packets. It is concluded that the AOMDV has better performance than the AODV. As the result of ability to find the alternate paths when the current paths fail, AOMDV is better than AODV.

Despite the fact that AOMDV causes higher routing overload, the packet delivery is more efficient than AODV. Therefore, it can be concluded that AOMDV could achieve better statistics in packet delivery than AODV and it could reduce the number of lost packets. Soo et al (2011) created the topology of Mobile Adhoc Network for this network and analyzed the influence of traffic flow on the energy consumption through applying AOMDV protocol compared with AODV. They also evaluated the parameters of performance such as the throughput, number of Packet loss, and End-to-End Delay. The characteristics of AOMDV and AODV performance include the workload analysis and the energy consumption level of nodes. The results of simulation indicate that the AOMDV has better performance than AODV in total workload according to the packet loss. The throughput of AOMDV is increased in low and average workload, but it is decreased in high workload. The End-to-end Delay is another important issue raised while applying the AOMDV and the use of larger number of hops can lead to the further end-to-end delay. According to the weaknesses of approach in this paper, the throughput of AOMDV is reduced in high workload and the large number of hops leads to the greater End-to-end Delay.

\section{Proposed Method}

A Fuzzifier is utilized for detecting the accuracy of an input variable. In this regard, a fuzzy set with a membership function is applied for indicating the degree of accuracy of set belonged to the linguistic variable. An input variable is converted to a single phase in the fuzzy interface system through a Fuzzifier and is mapped to the corresponding membership functions. The fuzzy operation acts on the input of fuzzy rule base of fuzzy interface system. A defuzzifier is a function to map the output of system from fuzzy to true domain. There are 2 special fuzzy models (Mamdani and Sugeno). These two models are different based on their output production techniques. Mamdani fuzzy interface system utilizes the defuzzification for producing the fuzzy output, but Sugeno uses the average weight to achieve the same purpose. The proposed algorithm of AOMDV-F in this research is combined with Sugeno fuzzy model.

The proposed algorithm is designed based on two parameters including the energy of path nodes and hop count in path. We extracted 24 fuzzy rules from these two parameters and decided according to them to select which paths from the available paths. 8 modes (Very Weak, Weak, Very Low, Low, Medium, Very High, High, and Full) were considered for the energy of nodes and three modes for the hop count (Low, Medium, High) and the fuzzy rules governing them are described as follows. The AOMDV routing protocol is applied in order to implement this algorithm. In this method, we have considered a field for calculating the average total energy of path and another field for the minimum energy of path. These two fields are sent along with the request packet and are updated while reaching each node. Then, the first node with the destination address adds these two fields to the response packet and disseminates it for the source. Since it is possible that the response sender node 
is not the destination, we disseminate the request packet until it reaches the destination. The reason is because we need to utilize the total energy of nodes. We then choose the path after doing the necessary operation and applying the proposed Fuzzy rules, and we disseminate the packets in selected path and continue this practice until the node with the lowest energy in the path reaches a minimum. At this time, we re-implement the routing to find a more efficient path than the previous path. According to the strategy of this algorithm, we continue this operation until $20 \%$ of all nodes in the network are diminished.

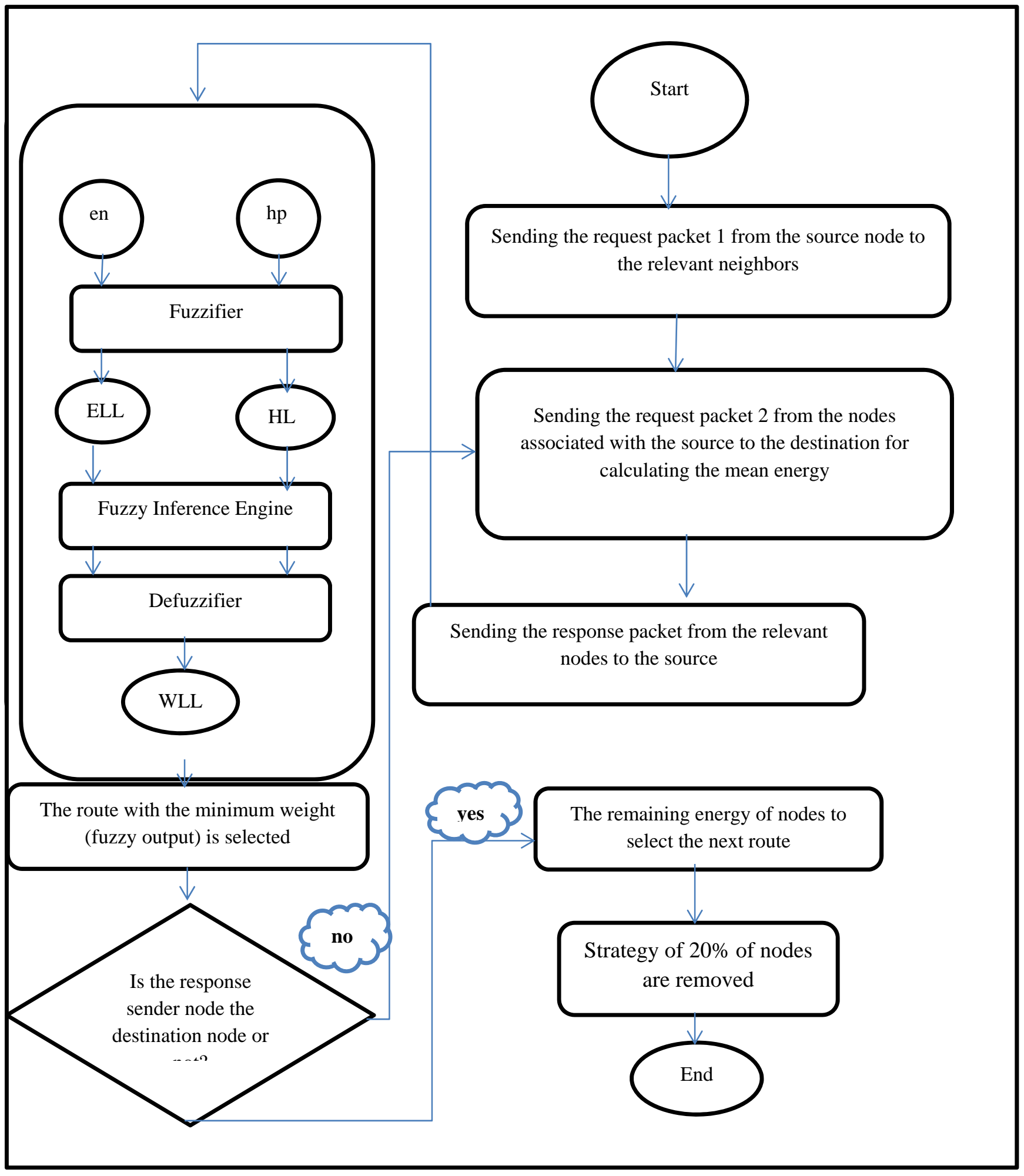

Fig. 1. Flowchart of proposed algorithm 


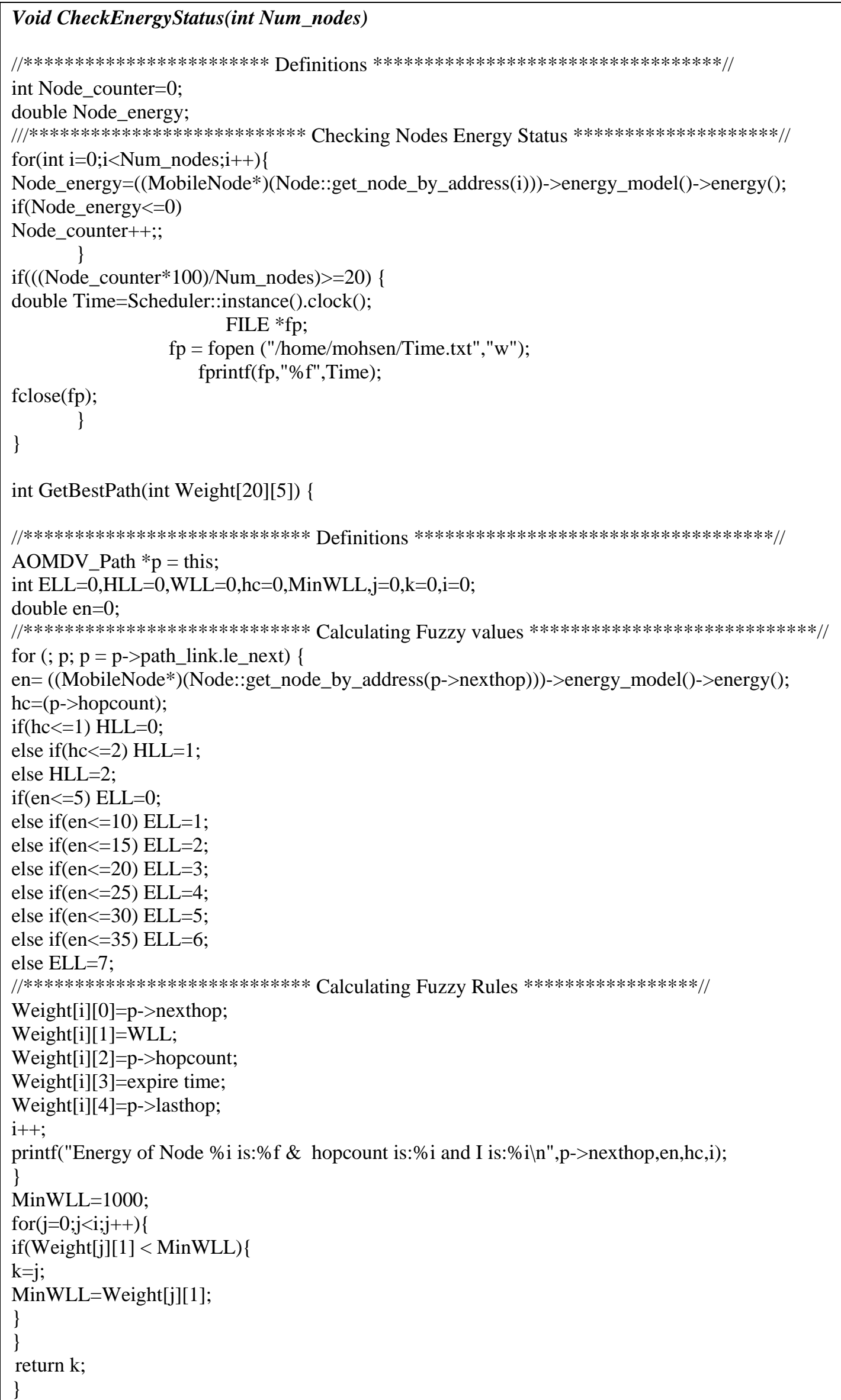

Fig. 2. The head file of proposed protocol AOMDV-F 
To find the optimal path, we consider an array, called "Weight" with the dimensions of $5 \times 20$, as the input parameter of function "Get Best Path". The column 0 in this matrix represents the next hop, Column 1 represents the path weight (WLL), which is the output of fuzzy system, Column 2 the hop count, Column 3 the expire time and Column 4 the last hop. We utilized the fuzzy logic to determine the weight value of each path because these both two parameters, energy of each node and the hop count, have the ambiguities along with each other.

If we consider using the path with the low hop count and higher energy, the network terminates its operation and fails due to the heavy use of selected path. In other words, the longevity of networks is reduced. Therefore, two parameters above should be combined by the Fuzzy Logic in a way that the longevity of network is increased by saving the energy consumption through selecting the optimal paths. We utilized the definition of functions in NS2 simulator software in order to implement the fuzzy method.

We applied Sugeno fuzzy logic described in the topics above. NS2 Software enables us to implement and edit the fuzzy inference system. This system has the input parameters: 1- amount of energy per node, 2- Hop count, and the output parameter of the path weight. The summary of the Fuzzy inference system is shown in Fig. 3.

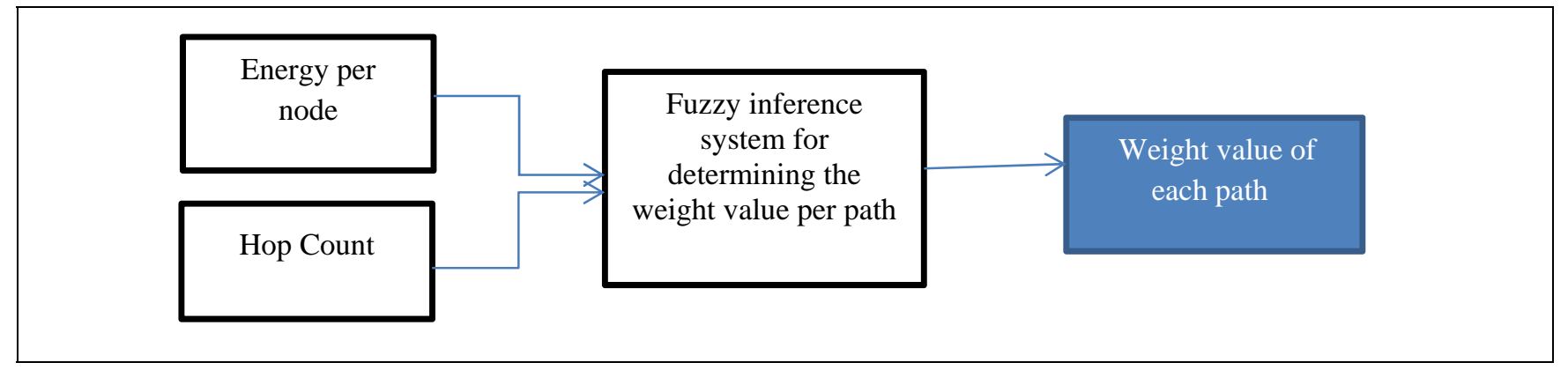

Fig. 3. The abstract view of fuzzy inference system for determining the weight value of each path

The output of fuzzy system in the proposed WLL algorithm represents the weight of path. The more the WLL is reduced, the more its energy is enhanced and has higher priority in routing process.

\subsection{Fuzzy Inference System Parameters}

\subsubsection{First fuzzy input of path value: The amount of energy per node}

We have shown the energy per node on the path (en) in the Fuzzy system with parameter ELL. The value of this input parameter is between 0 and 7 and has 8 modes discussed in the issues above. The more the interval related to this fuzzy parameter is reduced, the more it shows the accurate display of routing in the network.

\subsubsection{Second fuzzy input of path value: Hop Count}

The hop count (hc) or "the number of available nodes per path except for the source and destination) in Fuzzy system is shown with the parameter HLL. The value of this input parameter will be from 0 to 2 which include 3 modes. 
Table 3

Fuzzy Inference System parameters for determining the value weight per path

\begin{tabular}{|c|c|c|c|c|}
\hline \multirow{2}{*}{$\begin{array}{l}\text { Parameter } \\
\text { name }\end{array}$} & \multirow{2}{*}{ Type } & \multicolumn{3}{|c|}{ Membership function } \\
\hline & & Domain & Parameters & Domain \\
\hline \multirow{8}{*}{ ELL } & \multirow{8}{*}{ Input } & \multirow{8}{*}{ [0 7] } & {$\left[\begin{array}{lll}-1 & 0 & 0.9\end{array}\right]$} & Very Weak \\
\hline & & & [1 1.45 1.9] & Weak \\
\hline & & & [2 2.45 2.9] & Very Low \\
\hline & & & {$\left[\begin{array}{llll}3 & 3.45 & 3.9\end{array}\right]$} & Low \\
\hline & & & [4 4.45 4.9 ] & Medium \\
\hline & & & [5 5.45 5.9] & High \\
\hline & & & [ $\left.\begin{array}{lllll}6 & 6.45 & 6.9\end{array}\right]$ & Very High \\
\hline & & & [7 7.45 7.9] & Full \\
\hline \multirow{3}{*}{ HLL } & \multirow{3}{*}{ Input } & \multirow{3}{*}[\begin{array}{ll}{0}&{2}\end{array}]{} & {$\left[\begin{array}{lll}-1 & 0 & 0.9\end{array}\right]$} & Low \\
\hline & & & [1 11.45 1.9] & Medium \\
\hline & & & [2 2.45 2.9] & High \\
\hline \multirow{4}{*}{ WLL } & \multirow{4}{*}{ Output } & \multirow{4}{*}{ [10 240] } & {$\left[\begin{array}{llll}10 & 35 & 60\end{array}\right]$} & Full \\
\hline & & & [59 89 120] & High \\
\hline & & & [119 149.5 180] & Medium \\
\hline & & & [179 209.5240 [ & Low \\
\hline
\end{tabular}

ELL Parameter, which represents the energy of nodes, has 8 modes and HLL, which is the hop count, has 3 modes and 24 fuzzy rules ate extracted from combining these two parameters as shown in Table 4. ELL Parameter, which represents the energy of nodes, has 8 modes and HLL, which is the hop count, has 3 modes and 24 fuzzy rules are extracted from combining these two parameters as shown in Table 4. (It should be noted that 8 modes are selected for ELL parameter due to the higher accuracy in obtaining the results, so that the changes during routing are tangible).

For instance, we describe the sample first phase rule:

1) If (ELL is Very Weak) and (HLL is High) then (Wight is Low).

This rule states that if the "energy of node" is very weak and the "hop count during the path" is high, then the "path weight" will be low.

Table 4

The fuzzy rules of set

\begin{tabular}{cccc}
\hline RULE & ELL If ... and ... and ... & HLL & Then ... \\
\hline 1 & Very Weak & High & Low \\
2 & Very Weak & Medium & Low \\
3 & Very Weak & Low & Low \\
4 & Weak & High & Low \\
5 & Weak & Medium & Low \\
6 & Weak & Low & Low \\
7 & Very Low & High & Medium \\
8 & Very Low & Medium & Medium \\
9 & Very Low & Low & Medium \\
10 & Low & High & Medium \\
11 & Low & Medium & Medium \\
12 & Low & Low & Medium \\
13 & Medium & High & High \\
14 & Medium & Medium & High \\
15 & Low & High \\
16 & Medium & High & High \\
17 & High & Medium & High \\
19 & High & Low & High \\
\hline 20 & High & High & Full \\
\hline 21 & Very High & Medium & Full \\
\hline 23 & Very High & Low & Full \\
\hline
\end{tabular}




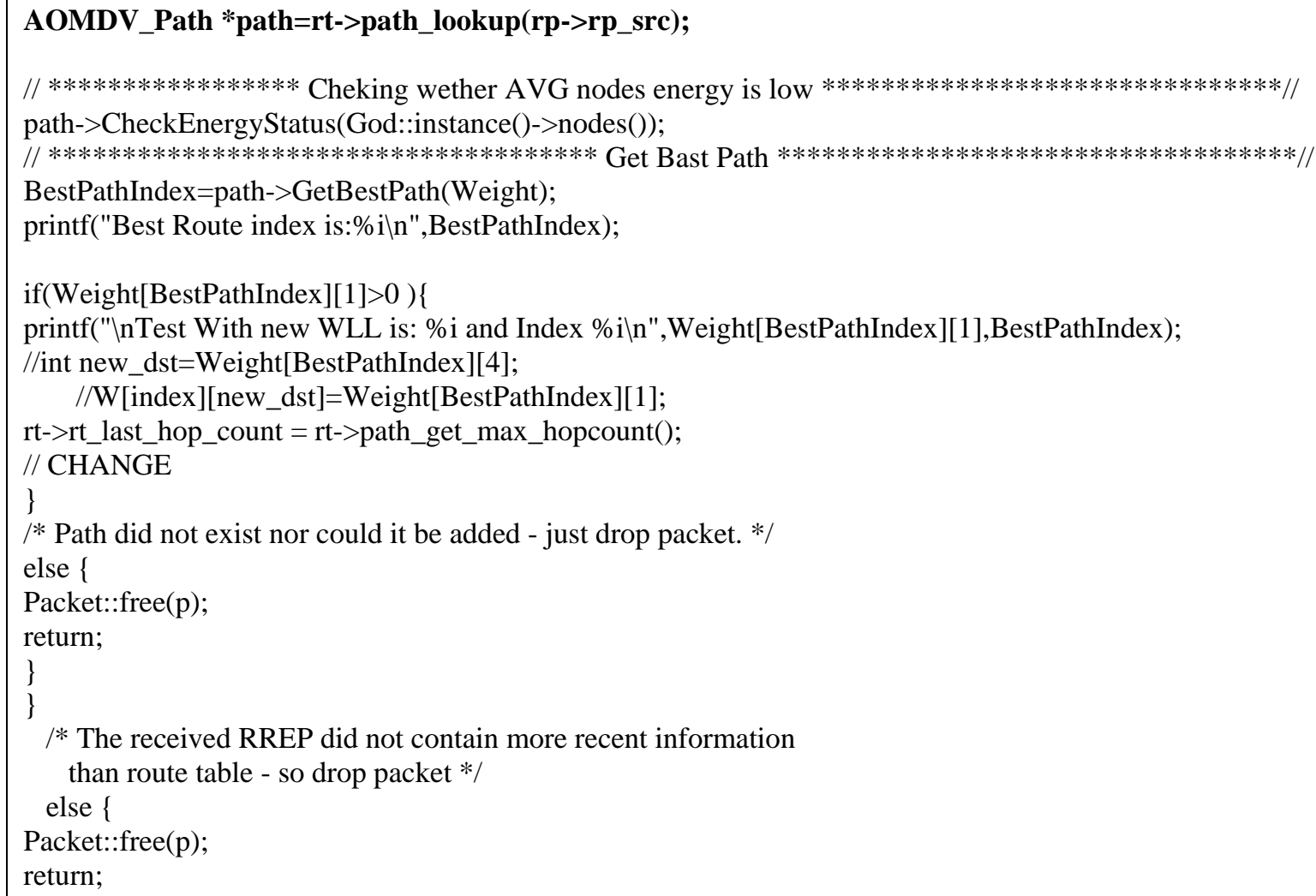

Fig. 4. The path searching algorithm in proposed protocol of AOMDV-F algorithm

We should determine the remaining energy of all nodes during the path because the nodes during the path lose some of their energies. Therefore, new forward path, which is better than the last one, is replaced.

\subsubsection{Final fuzzy output: Value weight of each path}

WLL is the output fuzzy system in the proposed algorithm and represents the weight of path. WLL is the Column (2) of weight matrix and shows the last optimal path. The minimum WLL in Weight Matrix represents the energy of that path, and finally the Index (k) displays the row number of matrix. We have assumed the minimum amount of weight equal to 1000 as a default; now if a row has the amount lower than the minimum weight, the relevant index will indicate that row.

\subsection{Determining the strategy of algorithm AOMDV-F}

As the result of the first node failure, the time begins for the failure of network; this algorithm seeks to retain the total network, so that the energy of available nodes in the network is properly applied.

The strategy in the proposed algorithm is in a way that if $20 \%$ of all available nodes in the network lose their nodes, then the network fails and thus the durability of network is increased.

\section{Simulation and its result}

The software packet of computer network simulator (NS-2) Version 2 is applied for simulation and evaluation of the proposed algorithm performance. This software is written by programming 
languages $\mathrm{C}++$ and OTCL. OTCL Language as an intermediary and command translator is utilized in designing the Simulator ns2.

Table 5

General Parameters of Simulation

\begin{tabular}{cc}
\hline Parameter & Amount \\
\hline Routing protocols & AODV, AOMDV \\
MAC Protocol & Mac/802_11 \\
Bandwidth / frequency & 0.4 Mbps \\
Simulation dimensions & $1000 \times 1000$ \\
Antenna type & Antenna/OmniAntenna \\
Transmission range & $200 \mathrm{~m}$ \\
Simulation time (milliseconds) & 500 \\
Number of nodes & 25 \\
Packet size & 1500 \\
\hline
\end{tabular}

\subsection{Performance Evaluation}

For evaluating the network performance, the changes are considered on three protocols AODV, AOMDV, AOMDV-F. The performance evaluation criteria in these three protocols are: The packet delivery rate, end-to-end delay time, and network throughput.

\subsubsection{End to end delay time}

It refers to the total delay of data packets to the destination and the dissemination, processing, transmission and queuing delay. It is better to have its higher amount in the source because the higher numbers of packets will be disseminated from the source and received at the destination. When the network size is small, AODV Protocol shows the lower delay than AOMDV and AOMDV-F, but AOMDV-F protocol will be improved significantly when the number of network nodes goes beyond 20 nodes. This is because this protocol is combined with fuzzy logic, thus it is less likely that the link fails; hence, the frequency resulted from the path maintenance is reduced. When the first selected path is retained, the link can lasted for a longer time, thus the frequency of path discovery is reduced and delay time is also decreased.

\subsubsection{Network Throughput}

The network throughput is the rate of data received at the destination node.Protocol AOMDV-F show further improvement than other two protocols especially when the network size is from 10 to 70.AOMDV-F shows better performance than AOMDV in most of cases and this is due to the choice of the most appropriate path and the relevant selected path is applied in the network for a longer term and there is a less need for retransmission of packets.

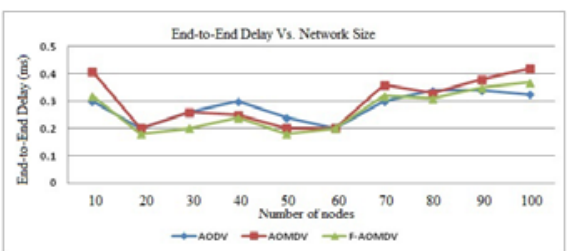

Fig. 5. Performance assessment Vs end to end delay

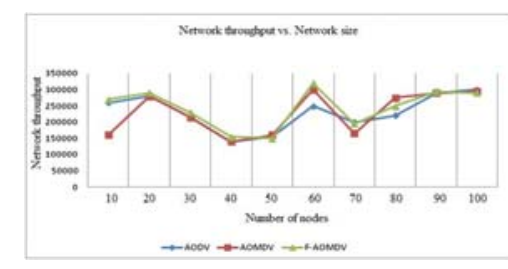

Fig. 6. Performance assessment vs. Network throughput

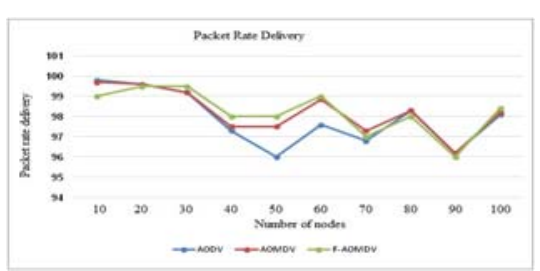

Fig. 7. The performance assessment vs. Packet Delivery Rate

\subsubsection{Packet delivery rate}

It refers to the number of delivered data disseminated from the source nodes. The results of simulation indicate that when the network size is from 10 to 20, AODV Protocol has better 
performance than other two protocols, but it depends on when the network size is small because the packet delivery rate is gradually decreased by increasing the size of network.

Therefore, the chance of losing the available paths is reduced among the nodes and data packets.

\section{Conclusion and future works}

\subsection{Obtained results}

The problems raised in wireless Ad hoc networks are as follows:

- Selecting the optimal path

- Selecting the path with the greater stability in the network

- Selecting the routing protocol

- The amount of lost data packets

- Hop count

- Energy Consumption

The durability of network is one of the main objectives in wireless ad hoc networks because the node is constantly moving and the energy, which is the important resource in such these networks, is reduced over time and the network ends its work at the less time. Therefore, the optimal routing of network in the network and the proper use of energy, which leads to the increases longevity and in other words, increased durability of network, are significantly important. When a node is lost in the network, the whole network fails, thus we will encounter the problems if we always apply the highenergy nodes and path with the lowest hop. According to the first problem, as the result of successive application of high-energy nodes, the level of energy in these nodes will be reduced faster and thus the network will fail.

Another problem is that the paths with higher hop count are not suitable because they increase the routing time and lead to the energy loss. Thus, these two parameters should be combined based on Fuzzy logic. AOMDV protocol, which is combined with the Fuzzy logic and has been able to resolve the problems above to some extent, are applied in the proposed has algorithm. AOMDV-F Protocol is the modified protocol based on the fuzzy logic. AOMDV Protocol has better performance than AODV in routing and it is capable of suggesting several paths instead of selecting and offering a path; in other words, it is a multi-path routing protocol. Two parameters, the energy of path nodes and the hop count of path, are considered in this study and 24 fuzzy rules are extracted by combining these two parameters for selecting the best path from the available paths. Out proposed algorithm is evaluated and compared on three protocols, AODV, AOMDV, and AOMDV-F, in NS-2 simulator in three criteria including the end-to-end delay performance, throughput of network and the packet delivery rate, and the results of these comparisons express the fact that the proposed protocol AOMDV-F has better performance than other protocols.

\subsection{Future Works}

- Given the improved performance of AOMDV-F protocol, it is predicted that if the numbers of fuzzy parameters are increased and another parameter such as the priority of delivery packets are combined in fuzzy logic, the routing accuracy will be significantly enhanced in the larger networks with higher numbers of nodes.

- The scheduling algorithms like FCFS, LRU, ... should be applied and the scheduling conditions of these algorithm in the proposed algorithm should be implemented under the fuzzy conditions in order to save the routing time. 


\section{References}

Bhavsar, C., Vegad, M. M., \& Bakhru, S. A. (2012). Performance Comparison of Stability Enhanced AODV and AOMDV Protocols for MANETs.. International Journal of Emerging Technology and Advanced Engineering. 2(5), 279-284.

Biradar, S. R., Majumder, K., Sarkar, S. K., \& Puttamadappa, C. (2010). Performance evaluation and comparison of AODV and AOMDV. International Journal on Computer Science and Engineering, 2(02), 373-377.

Boukerche, A. (Ed.). (2008). Algorithms and protocols for wireless, mobile ad hoc networks (Vol. 77). John Wiley \& Sons.

Chiang, M. (2005). Balancing transport and physical layers in wireless multihop networks: Jointly optimal congestion control and power control. Selected Areas in Communications, IEEE Journal on, 23(1), 104-116.

ElBatt, T., \& Ephremides, A. (2004). Joint scheduling and power control for wireless ad hoc networks. Wireless communications, IEEE Transactions on,3(1), 74-85.

IEEE Standard 802.11e.Part11: Wireless LAN medium access control (MAC) and physical layer (PHY) specifications. Amendment 8: Medium Access Control(MAC) (2005). Quality Of Service(QOS), IEE, 1-212.

Jia, L., Liu, X., Noubir, G., \& Rajaraman, R. (2005, March). Transmission power control for ad hoc wireless networks: throughput, energy and fairness. InWireless Communications and Networking Conference, 2005 IEEE (Vol. 1, pp. 619-625). IEEE.

Johansson, M., \& Xiao, L. (2004, May). Scheduling, routing and power allocation for fairness in wireless networks. In Vehicular Technology Conference, 2004. VTC 2004-Spring. 2004 IEEE 59th (Vol. 3, pp. 1355-1360). IEEE.

Jung, E. S., \& Vaidya, N. H. (2005). A power control MAC protocol for ad hoc networks. Wireless Networks, 11(1-2), 55-66.

Kawadia, V., \& Kumar, P. R. (2005). Principles and protocols for power control in wireless ad hoc networks. Selected Areas in Communications, IEEE Journal on, 23(1), 76-88.

Kawadia, V., \& Kumar, P. R. (2005). A cautionary perspective on cross-layer design. Wireless Communications, IEEE, 12(1), 3-11.

Lai, W. K., Weng, M. L., \& Lin, Y. H. (2012). Improving MANET performance by a hop-aware and energy-based buffer management scheme. Wireless Communications and Mobile Computing.

Muqattash, A., \& Krunz, M. (2005). POWMAC: a single-channel power-control protocol for throughput enhancement in wireless ad hoc networks. Selected Areas in Communications, IEEE Journal on, 23(5), 1067-1084.

Perkins, C. E., \& Royer, E. M. (1999, February). Ad-hoc on-demand distance vector routing. In Mobile Computing Systems and Applications, 1999. Proceedings. WMCSA'99. Second IEEE Workshop on (pp. 90-100). IEEE.

Radunovic, B., \& Le Boudec, J. Y. (2005, June). Power control is not required for wireless networks in the linear regime. In World of Wireless Mobile and Multimedia Networks, 2005. WoWMoM 2005. Sixth IEEE International Symposium on a (pp. 417-427). IEEE.

Radunovic, B., \& Le Boudec, J. Y. (2004). Optimal power control, scheduling, and routing in UWB networks. Selected Areas in Communications, IEEE Journal on, 22(7), 1252-1270.

Soo, A. L., Tan, C. E., \& Tay, K. M. (2011, June). Fuzzy multiple metrics link assessment for routing in mobile Adhoc network. In AIP Conference Proceedings, 1337, 149.

Haque, I. T., \& Assi, C. (2007). Localized energy efficient routing in mobile ad hoc networks. Wireless Communications and Mobile Computing, 7(6), 781-793. 